\title{
Correlations Between Severity of Coronary Lesions and Epicardial Fat Volume in Patients with Coronary Artery Disease - a Multislice CT-based Study
}

\author{
Roxana Hodaș, Sorin Pop¹, Diana Opincariu², Nora Rat', Laura Jani², Alexandra Stanescu², Edvin \\ Benedek², Imre Benedek1, Theodora Benedek', Monica Chitu' \\ ${ }^{1}$ Clinic of Cardiology, University of Medicine and Pharmacy, Tîrgu Mureș, Romania \\ 2 Center of Advanced Research in Multimodality Cardiac Imaging, Cardio Med Medical Center, Tîrgu Mureș, Romania
}

\section{CORRESPONDENCE}

\section{Sorin Pop}

38 Gheorghe Marinescu St

540139 Tîrgu Mureș, Romania

Tel: 0265215551

Email: sorinp91@yahoo.com

\section{ARTICLE HISTORY}

Received: 15 April, 2016

Accepted: 16 May, 2016

Roxana Hodaș • 38 Gheorghe Marinescu St, 540139 Tîrgu Mureș, Romania, Tel: +40 265215551 Email: roxana.hodas@yahoo.ro.

Diana Opincariu • 76, 22 Decembrie 1989 St, 54012 Tîrgu Mureș, Romania, Tel: +40 265217333 Email: diana.opincariu@yahoo.ro

Nora Rat • 38 Gheorghe Marinescu St, 540139 Tîrgu Mureș, Romania, Tel: +40 265215551

Email: ratnora@gmai.com

Laura Jani • 76, 22 Decembrie 1989 St, 540124 Tîrg Mureș, Romania, Tel: +40 265217333

Email: jlaura15@yahoo.com

Alexandra Stanescu • 76, 22 Decembrie 1989 St, 540124 Tîrgu Mureș, Romania, Tel: +40 265217333 Email: alexandrastanescu90@gmail.com

Edvin Benedek • 76, 22 Decembrie 1989 St, 540124 Tîrgu Mureș, Romania, Tel: +40 265217333 Email: edvinke87@gmail.com

Imre Benedek • 38 Gheorghe Marinescu St, 540139 Tîrgu Mures, Romania, Tel: +40 265215551 Email: imrebenedek@yahoo.com

Theodora Benedek • 38 Gheorghe Marinescu St, 540139 Tîrgu Mures, Romania, Tel: +40 265215551 Email: theodora.benedek@gmail.com

Monica Chitu • 38 Gheorghe Marinescu St, 540139 Tîrgu Mures, Romania, Tel: +40 265215551

Email: iuliachitu@yahoo.com

\section{ABSTRACT}

Background: Epicardial fat has been recently identified as a major player in the development of the atherosclerotic process. Study aim: The aim of this study was to correlate the epicardial fat volume (EFV), determined by Multisclice CT, and the severity of the coronary lesions, expressed by the Coronary Calcium Score (CCS) and Syntax Score (SxS) in patients with established coronary artery disease (CAD). Material and methods: One-hundred-twentysix patients underwent Multisclice 64 CT assessment of coronary lesions and epicardial fat quantification. Calculation of CCS was performed on all the three coronary vessels and was followed by determination of SxS according to guidelines. The patients were divided into 2 groups: Group 1 - patients with CCS $>400(n=26)$, and Group 2 - patients with CaS $<400$ (n $=100$ ). Results: The mean age of the study population was 65.32 years for Group 1 and 54 years for Group 2 ( $p<0.0001$ ). However, patients $>65$ years of age had a high CCS in a more significant extent than younger patients (50\% in Group 1 vs. 17\% in Group 2, p = 0.0115). Female gender was recorded in 48\% of cases in Group 2 and in 19\% of cases in Group 1 ( $p=0.008$ ). Several factors were identified in a higher extent in the group with high CCS as compared with the group with low CCS, such as the presence of significant stenosis (>50\%) of the left anterior descending artery (LAD) (46\% vs. $9 \%, p<0.0001)$, the presence of multi-vessel coronary disease (50\% vs. 5\%, $p<0.0001)$ and a high SxS, above 23 (23\% vs. 4\%, p = 0.006). The epicardial fat volume was $117.81 \pm 40.4 \mathrm{ml}(95 \% \mathrm{Cl}$ : 97.98-138.2 ml) in Group 2 and $89.77 \pm 37.7 \mathrm{ml}(95 \%$ Cl: 80.4-101.5 ml) in Group 1 ( $p=0.0033)$. Conclusions: Epicardial fat volume could represent a new imaging-derived biomarker, useful for classification of the severity of coronary artery disease, increased values of EFV being associated with other biomarkers of disease severity, such as calcium score.

Keywords: epicardial fat volume, coronary artery disease, Multisclice CT, Calcium Score, Syntax Score 


\section{INTRODUCTION}

Epicardial adipose tissue is a metabolically active visceral fat deposit located around the heart, between the myocardium and the visceral pericardium, directly surrounding the subepicardial coronary vessels. Due to the absence of fascial boundaries, it is in close anatomical and functional relationship with both the myocardium and the adventitia of the coronary arteries. ${ }^{1,2}$

This tissue can adapt to different metabolic profiles by changing its cellular composition. Under physiological conditions, the epicardial adipose tissue displays biochemical, mechanical and thermogenic cardioprotective properties, but in pathological circumstances, many changes occur as the epicardial fat volume increases. ${ }^{3}$ These changes are often characterized by failure to store triglycerides, increased lipolysis, hypertrophy and, most importantly, stimulation of inflammation. As epicardial fat expands, it becomes dysfunctional and invaded by macrophages and T cell lymphocytes. ${ }^{4,5}$

Because of proximity to the coronary arteries, the epicardial fat has recently been identified as a major role player in the development of the atherosclerotic process. It acts as an endocrine organ through several paracrine mechanisms of locally secreted adipocytokines and other bioactive molecules, but also through systemic pro-inflammatory effects on the cardiovascular system. Paracrine release of pro-inlammatory cytokines from the peri-adventitial adipose tissue could pass through the coronary wall and locally interact with the structures of each layer. It has been demonstrated that the adipose tissue participates in various stages of atherogenesis, from endothelial dysfunctions to plaque destabilization and rupture. ${ }^{6,7}$ Harmful and possible protective effects are in a state of imbalance, and its effects are still unknown. ${ }^{8}$

Previous studies have also demonstrated the association between the amount of epicardial adipose tissue and the severity of coronary artery disease (CAD), therefore, the non-invasive assessment of epicardial fat could represent a simple and reliable indicator of cardiovascular risk. ${ }^{1}$ In order to measure the amount of epicardial fat volume, currently three diagnostic tools are in use: echocardiography, multi-slice computed tomography (MSCT) and magnetic resonance imaging (MRI).

Two-dimensional (2D) echocardiography can visualize and measure the epicardial fat thickness on the right ventricular free wall from both parasternal long-axis and shortaxis views. But it is well known that the epicardial adipose tissue is not evenly distributed around the heart and this is a linear measurement at a single location, thus, it may not reflect the variability of fat tissue thickness or total fat volume. ${ }^{9}$ As recently suggested, rather than its thickness, the epicardial fat volume (EFV) may be the most consistent measure of the cardiovascular risk. Its advantages are the large availability and the less expensive cost as compared with MRI or CT. ${ }^{10}$

It is a well-known fact that MRI is the gold standard for the body adipose tissue quantification. Recent studies have used cardiac MRI as a tool in order to measure the epicardial adipose tissue by using the summation of slices method. There is a good correlation between the measurements obtained on echocardiography and cardiovascular magnetic resonance imaging (CMR). Although the image resolution and accuracy is better on MRI, as compared with CT, currently MRI still remains limited, due to its high costs and low accessibility. ${ }^{11,12}$

Recent evidence is suggesting that the EFV measured on MSCT may have a predictive value for coronary artery disease (CAD). ${ }^{13}$

MSCT, unlike other imaging methods, is capable of simultaneously visualizing and quantifying the coronary atherosclerotic burden through the Coronary Calcium Score (CCS) and the Syntax Score (SxS), and also the total amount of epicardial adipose volume. Furthermore, MSCT provides more objective results and a more accurate evaluation of the adipose tissue because of its higher spatial resolution compared to the echocardiographic assessment. ${ }^{14}$

\section{STUDY AIM}

The aim of this study was to correlate the epicardial fat volume, determined by Multisclice CT, and the severity of coronary lesions expressed by the CCS and the SxS, in patients with clinical suspicion of coronary artery disease.

\section{MATERIAL AND METHODS}

\section{Study population}

We retrospectively included in our study 126 patients with clinical suspicion of CAD, who were clinically reffered for non-invasive Multislice Computed Tomography (MSCT), coronary angiography for CAD evaluation betweeb January 2015 and December 2015. Patients were divided into 2 groups on the basis of CCS: Group $1-26$ patients with high CCS $(>400)-$ high risk, and Group $2-100$ patients with low CCS $(<400)-$ low risk. The study has been carried out in accordance with the code of ethics of the World Medical Association's Declaration 
of Helsinki. All patients gave informed consent, and the study protocol was approved by the ethics committee of the Cardio Med Medical Center, the center where the study was conducted.

\section{Risk factor assessment}

The patients' demographic characteristics, medical history and traditional coronary risk factors were obtained from medical reports. Routine biochemistry results including lipid profile, liver enzymes, inflammatory status, urea, creatinine and full blood count were also recorded.

Hypertension was defined as a systolic blood pressure $>140 \mathrm{mmHg}$, a diastolic blood pressure $>90 \mathrm{mmHg}$ or antihypertensive medication. Dyslipidemia was classified as a total cholesterol (TC) $>200 \mathrm{mg} / \mathrm{dl}$, low-density lipoprotein (LDL) cholesterol $>130 \mathrm{mg} / \mathrm{dl}$, high-density lipoprotein (HDL) cholesterol $<30 \mathrm{mg} / \mathrm{dl}$ or treatment with lipid-modifying agents. Diabetes mellitus was defined as a fasting glucose $>126 \mathrm{mg} / \mathrm{dl}$ on two or more occasions, or hypoglycemic treatment, or if they had been previously diagnosed.

\section{Echocardiographic Measurement}

Each patient underwent transthoracic echocardiography. Ejection Fraction (EF), LVED volume, LVES volume, $\mathrm{E} / \mathrm{A}$ ratio and $\mathrm{LV} \mathrm{dP} / \mathrm{dt}$ were measured in each patient.

\section{Coronary CT imaging protocol}

All patients underwent a 64-slice cardiac CT scan in order to visualize and quantify the coronary atherosclerotic burden. Patients with uncontrolled heart rate $(>65$ bpm) received oral beta blocker (metoprolol $50 \mathrm{mg}$ ). The Epicardial Fat Volume (EFV) and Coronary Artery Calcification (CAC) were obtained using a standard scanning protocol. In non-contrast cardiac CT the total calcium burden of the coronary tree was determined by semiautomated analysis using the Agatstonscore (CCS). $\mathrm{EFV}$ was also quantified by calculating the total sum of epicardial adipose tissue areas measured from $1 \mathrm{~cm}$ above the left main coronary artery to the left ventricular apex, in $1 \mathrm{~cm}$ intervals. The epicardial adipose thickness (EAT) was defined as adipose tissue surrounding the myocardium and limited by the epicardium. The pericardium was manually traced from the right pulmonary artery to the diafragm, all extra-pericardal tissue was excluded and fat voxels were identified using threshold attenuation values.

\section{Statistical analysis}

All data was analysed by using GraphPad Prism 6. Categorical variables were compared with Pearson's Chi-square test and are expressed as numbers and percent values (\%). Continuous data are presented as mean value \pm standard deviation, and were compared with Student's $t$ test for variables with gaussian distribution and Mann-Whitney U test for variables with non-gaussian distribution.

Univariate linear regression analysis was performed in order to investigate the association of EFV with CCS and Syntax Score. In our analysis, a p value of less than 0.05 was considered statistically significant.

\section{Coronary artery lesion assessment (Syntax Score calculation)}

In order to visualize the angiographic characteristics, each patient underwent 64-slice cardiac CT, as previously mentioned. The coronary artery tree of each patient was accounted by using the Syntax Score (SxS). The SxS system consist of a set of sequential questions, in which every coronary lesion that generates $>50 \%$ luminal obstruction in vessels with a lumen $\geq 1.5 \mathrm{~mm}$ was separately scored and summated to provide the overall SYNTAX score. SxS was calculated using a dedicated software that integrates the following components: left or right dominance, total occlusion, whether bifurcation, trifurcation or aorta ostial lesion, severe tortuosity, heavy calcification, thrombus, diffuse disease, and diseased coronary artery segment longer than $20 \mathrm{~mm}$.

A total occlusion was defined as a lesion with an abrupt vessel cut-off. One or more bends of $90^{\circ}$ or more, or three or more bends of $45^{\circ}$ to $90^{\circ}$ proximal of the diseased segment was defined as tortuosity.

\section{RESULTS}

One hundred and twenty-six patients were enrolled in the study. Coronary calcium score was high in $20.63 \%$ of patients (Group 1, $\mathrm{n}=26$ ) and low in $79.36 \%$ of patients (Group 2, $\mathrm{n}=100$ ). The patient demographics and cardiovascular risk assessment are summarized in Table 1.

Patients with high a CCS were significantly older than those with a low CCS: the mean age of patients was 65.35 \pm 1.13 years in Group 1 and $54 \pm 1.16$ years in Group 2 (p $<0.0001)$.

However, patients $>65$ years of age had a high CCS to a more significant extent than younger patients $(50 \%$ in Group 1 vs. $17 \%$ in Group 2, $\mathrm{p}=0.0115$ ). 
TABLE 1. Patient demographics and cardiovascular risk factors

\begin{tabular}{|c|c|c|c|c|c|}
\hline & \multicolumn{2}{|c|}{ Group 1 (CCS >400) } & \multicolumn{2}{|c|}{ Group 1 (CCS <400) } & \multirow[t]{2}{*}{ p value } \\
\hline Number & 26 & & 100 & & \\
\hline Mean Age & $65.35 \pm 1.13$ & & $54.02 \pm 1.16$ & & $p<0.0001$ \\
\hline Patients > 65 yo & 13 & $50 \%$ & 17 & $17 \%$ & $p=0.0115$ \\
\hline Patients < 50 yo & 0 & ०\% & 37 & $37 \%$ & \\
\hline Men & 21 & $80.77 \%$ & 52 & $52 \%$ & \\
\hline Women & 5 & $19.23 \%$ & 48 & $48 \%$ & $p<0.0001$ \\
\hline Smokers & 2 & $7.69 \%$ & 20 & $20 \%$ & $p<0.0001$ \\
\hline Hypertension & 25 & $96.15 \%$ & 89 & $89 \%$ & $p=0.1046$ \\
\hline Diabetes mellitus & 5 & $19.23 \%$ & 16 & $16 \%$ & $p=0.7102$ \\
\hline Dyslipidemia & 14 & $53.85 \%$ & 57 & $57 \%$ & $p=0.7761$ \\
\hline Triglyceries, mg/dl & $157.01 \pm 1.12$ & & $151 \pm 0.56$ & & \\
\hline Mean cholesterol value & 217 & & 211 & & \\
\hline HDL cholesterol, mg/dl & $47.99 \pm 1.14$ & & $56.08 \pm 0.99$ & & \\
\hline
\end{tabular}

The female gender population was significantly larger in the low CCS group: $19 \%$ of cases in Group 1 and $48 \%$ of cases in Group 2 ( $\mathrm{p}=0.008)$.

With regard to the risk factors of $\mathrm{CAD}$, the lipid profiles including levels of total cholesterol, LDL, HDL and triglycerides were not significantly different in the two groups. Also, there were no statistically significant differences between the 2 groups regarding the presence of diabetes $(p=0.7102)$, dyslipidemia $(p=0.7761)$, arterial hypertension ( $\mathrm{p}=0.1046)$, but we observed that the low CCS group had a higher prevalence of smoking patients, compared to the other group ( $20 \%$ in Group 2 vs. $8 \%$ in Group 1, p <0.0001).

The mean value of left ventricular ejection fraction measured by echocardiography was significantly lower in the risk group (Group $1-47.72 \% \pm 1.28$ vs. Group $2-51.9 \%$ $\pm 0.57, \mathrm{p}=0.0020)$. Also, there were more patients with
LVEF values under $45 \%$ in Group 1 compared with Group 2 , but there was no significant difference ( $15 \%$ in Group 1 vs. $6 \%$ in Group 2, p = 0.0629) (Figure 1).

There were significant differences between the two groups in terms of angiographic characteristics, such as the presence of multivascular coronary artery disease and the severity of the stenosis. The presence of 2-vessel coronary artery disease was significantly higher in the high risk group ( $23 \%$ vs. $11 \%, \mathrm{p}=0.0080$ ). The 3 -vessel CAD was also more pronounced in the high CCS group compared to the other group $(50 \%$ vs. $5 \%, \mathrm{p}<0.0001)$ (Figure 2).

Regarding the presence of severe stenosis $(>70 \%)$ on each coronary artery, we obtained significantly higher values in the risk group. Severe LAD stenosis was present in $46 \%$ of patients in Group 1, compared to only $9 \%$ in Group 2 ( $p<0.0001)$ (Figure 3A). A severe circumflex artery ste-
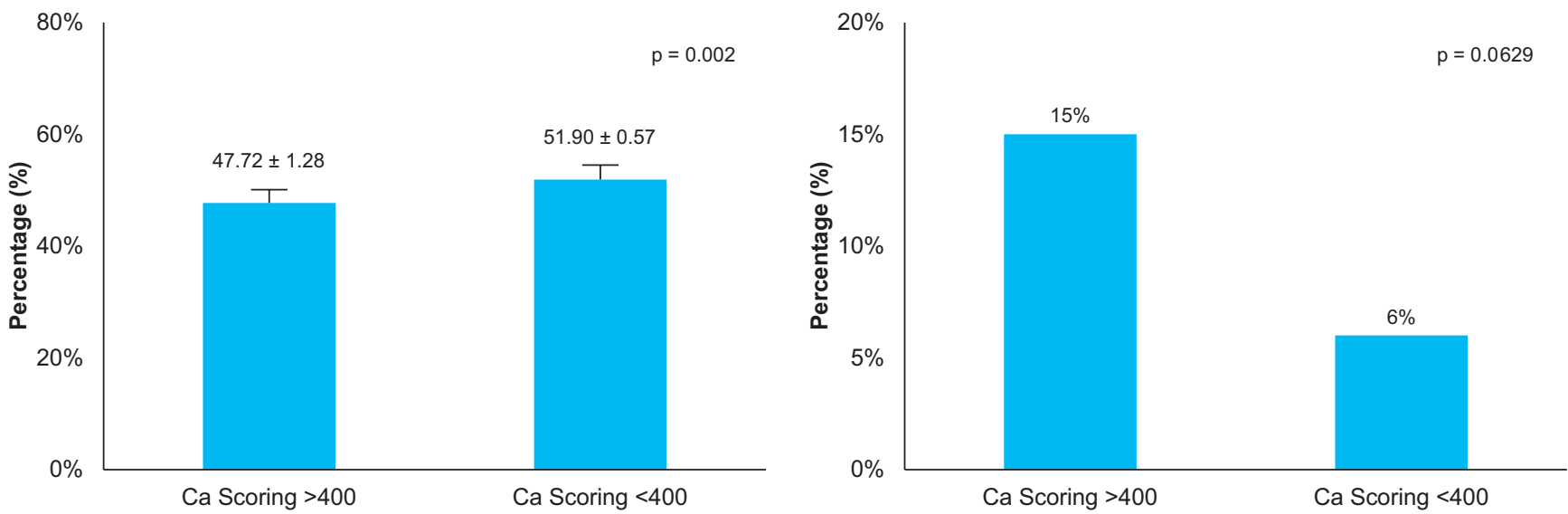

FIGURE 1. Left ventricular ejection fraction in the study groups. A - Mean values of EF; $\mathbf{B}-$ Patients with EF $<45 \%$ 


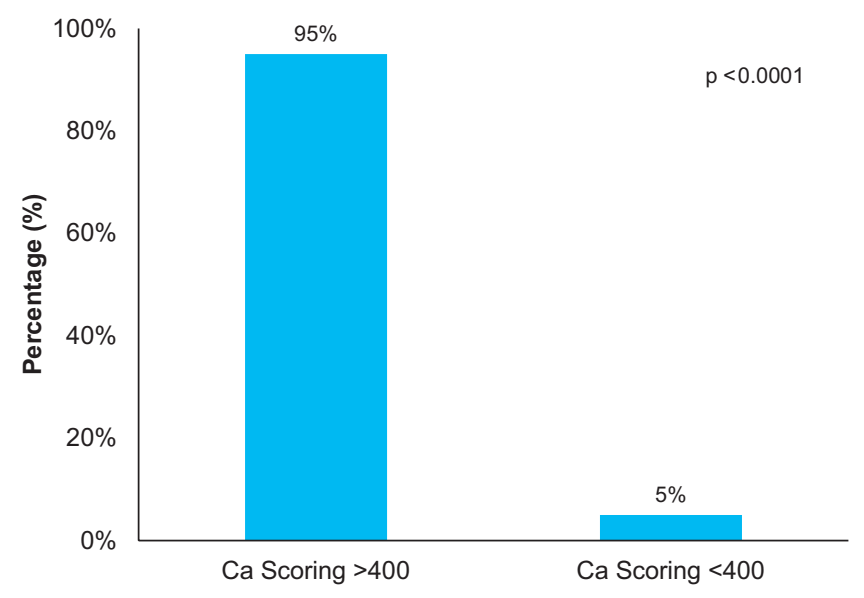

FIGURE 2. Presence of 3-vessel disease in the study groups

nosis was found in $19 \%$ of patients in Group 1, compared with only $2 \%$ in Group 2 ( $\mathrm{p}<0.0001)$ (Figure 3B) and there were $19 \%$ of patients with severe RCA stenosis in Group 1
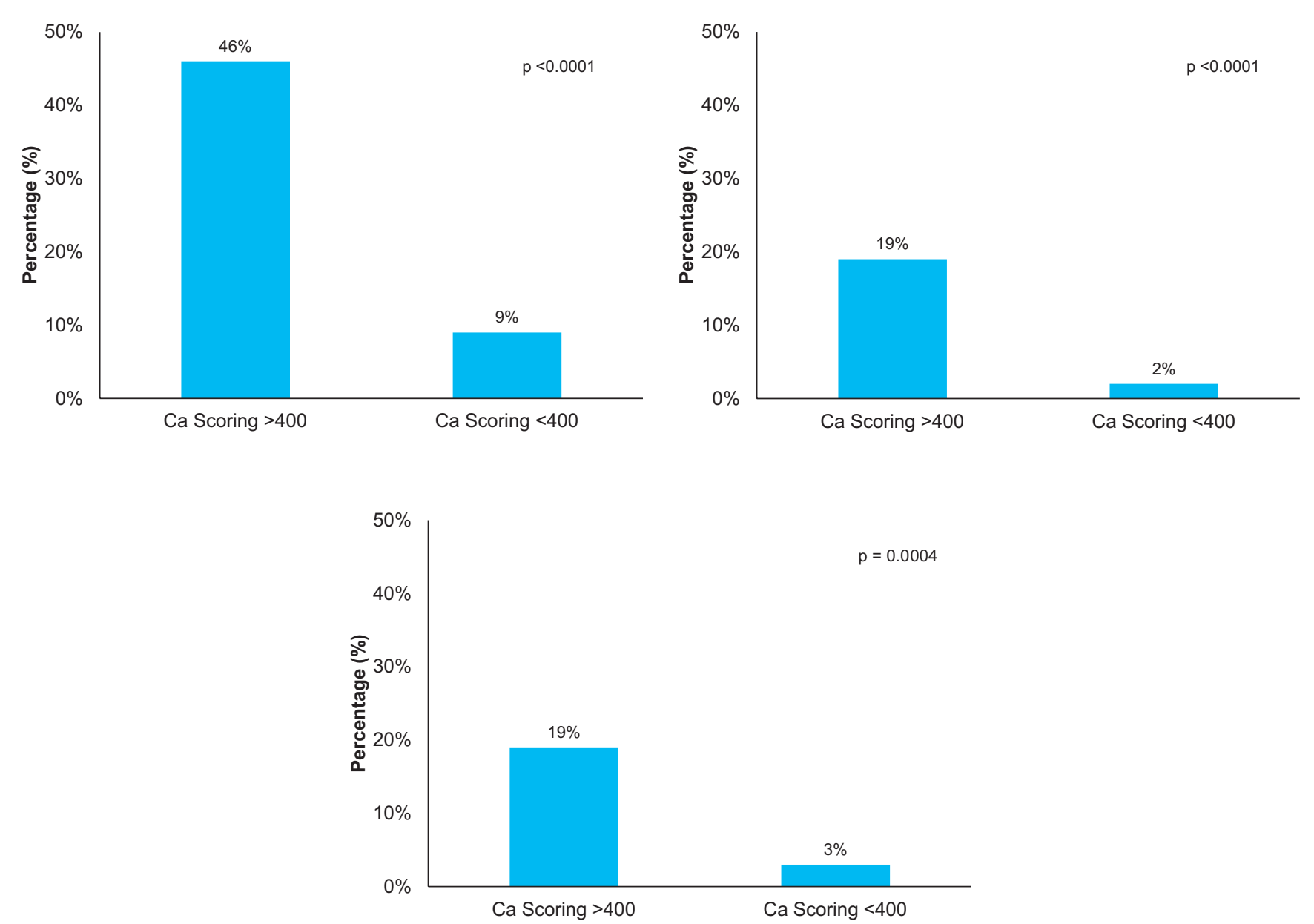

FIGURE 3. Angiographic aspects in the study groups. A - Stenoses located on the left anterior descendent artery (LAD); B - Stenoses located in the circumflex artery (CXA); $\mathbf{C}$ - Stenoses located in the right coronary artery (RCA) as compared with only $3 \%$ in Group $2(\mathrm{p}=0.0004)$ (Figure $3 \mathrm{C})$. These results show that a high CCS is significantly associated with severe coronary artery stenosis.

The mean epicardial fat volume, measured by MSCT, was $117.81 \pm 40.4 \mathrm{ml}$ (95\% CI: 97.98-138.2 ml) for Group 1 and $89.77 \pm 37.7 \mathrm{ml}$ (95\% CI: $80.4-101.5 \mathrm{ml})$ for Group 2 , the difference being highly statistically significant ( $\mathrm{p}=$ 0.0033) (Figure 4).

In regards to the Syntax Score values, the high CCS risk group showed significantly higher Syntax Score values $(39.64 \pm 15.64)$ compared to the other group (3.94 \pm 0.74 ) and also higher risk values ( $\mathrm{SxS}>23$ : Group 1: $23 \%$ vs. Group 2: 4\%) indicating a greater risk in the high CCS group (Figure 5).

The simple linear regression analysis demonstrated that the epicardial fat volume was well correlated with both the degree of coronary calcification, quantified by CCS ( $p=$ $0.0129, r=0.2406)$ and the Syntax Score $(p=0.0229, r=$ 0.2209 ) in CAD patients (Figure 6).

$$
p=0.0004
$$




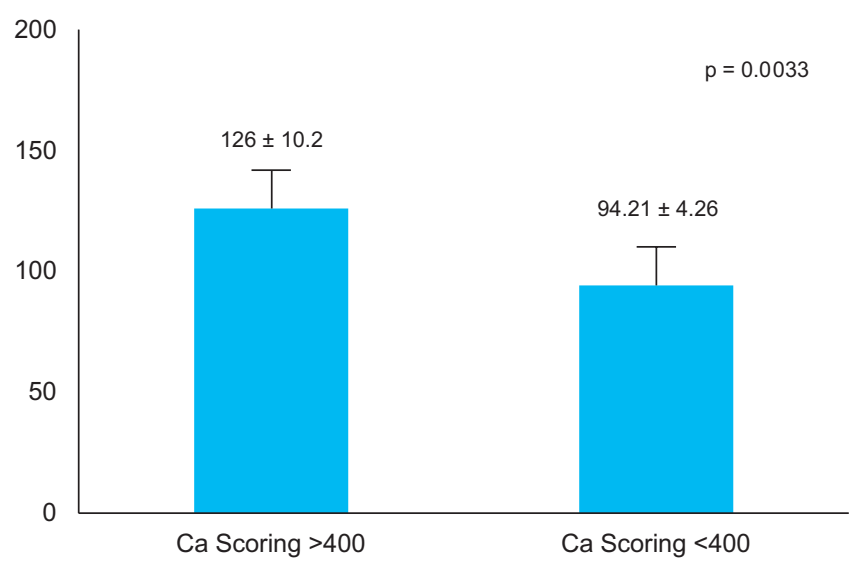

FIGURE 4. Epicardial fat volume in the study groups

\section{DISCUSSIONS}

In the present analysis, we described the connection between the EFV (measured by MSCT) and the CCS, and the $\mathrm{SxS}$ as markers of the coronary plaque burden. The diagnostic value of the EFV measured with MSCT was assessed in order to detect functionally relevant CAD.

Over the last years several studies have investigated a potential correlation between epicardial adipose tissue and coronary artery plaque burden, represented through different biomarkers given the biochemical properties of EAT (epicardial adipose thickness) and its possible role as a cardiovascular risk factor. ${ }^{15}$ An estimation of EAT would be important, hence several methods have been applied as a surrogate for its assessment. Overall non-invasive quantitative measurement of EFV on MSCT is feasible and could play a role in cardiovascular risk assessment. Its correlation to the presence of CAD, severity and prognosis have been shown by previous studies.

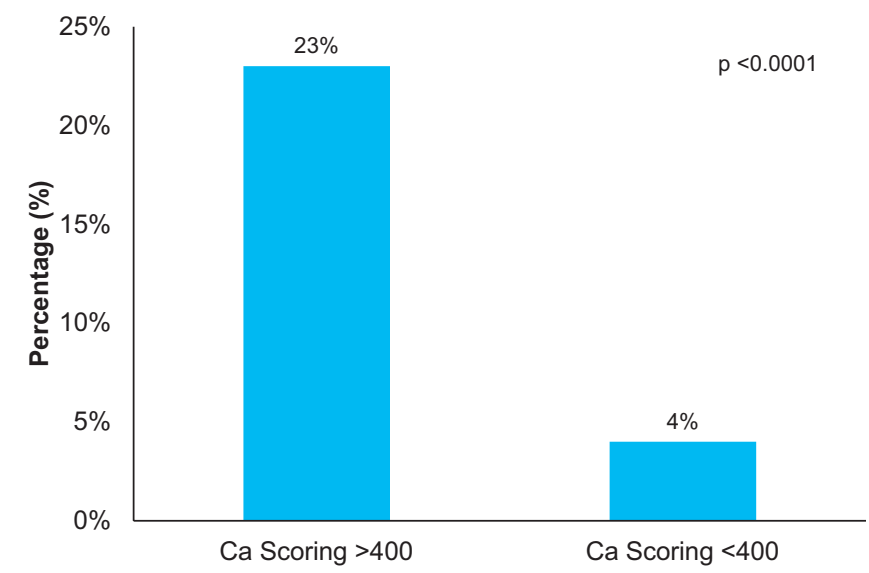

In this study we found sizeable differences in EFV, which was particularly high in the high-risk group. Patients with high CCS $(>400)$ showed significantly higher volumes of adipose tissue than those with low CCS $(<400)$. Secondly, EFV values were in good association with both the CCS and the SxS, which is in line with previous studies that have suggested that $\mathrm{EFV}$ is associated with significant CAD. Iwasaki et al. also observed, on a univariate analysis, a higher EFV in patients with significant coronary stenosis $(>50 \%)$ compared to patients without CAD. ${ }^{16}$ Also, Ueno et al. have shown that $\mathrm{EFV}$ is independently related to chronic total occlusions. ${ }^{17}$ The Framingham Heart Study found an association between EAT and CCS in a cross-sectional analysis, but only when including the adipose tissue in addition to traditional risk factors. This fact was explained in the Heinz Nixdorf Recall study by a shared risk factor profile of both EAT and CCS.18 These and other previous studies have shown the clinical significance of EFV measured with MSCT.

Nevertheless, a substantial part of the literature identifies a lack of correlation between EAT volume and CCS, these results supporting the hypothesis that epicardial fat is involved in the early stages of the coronary atherosclerotic process.

We have also shown that the extent of coronary artery calcification is associated with severe coronary stenosis $(>70 \%)$ on each coronary artery and with the presence of 3 -vessel disease; high CCS was associated with severe arterial stenosis and, there have also been reports that suggest that EFV itself is associated with coronary stenosis and plaque morphology on cardiac CT.

Interestingly, there were significantly more women in the low-risk group ( $\mathrm{CCS}<400)$, thus showing that female gender acts as a protective factor vis-à-vis the atherosclerotic process of the coronary arteries.

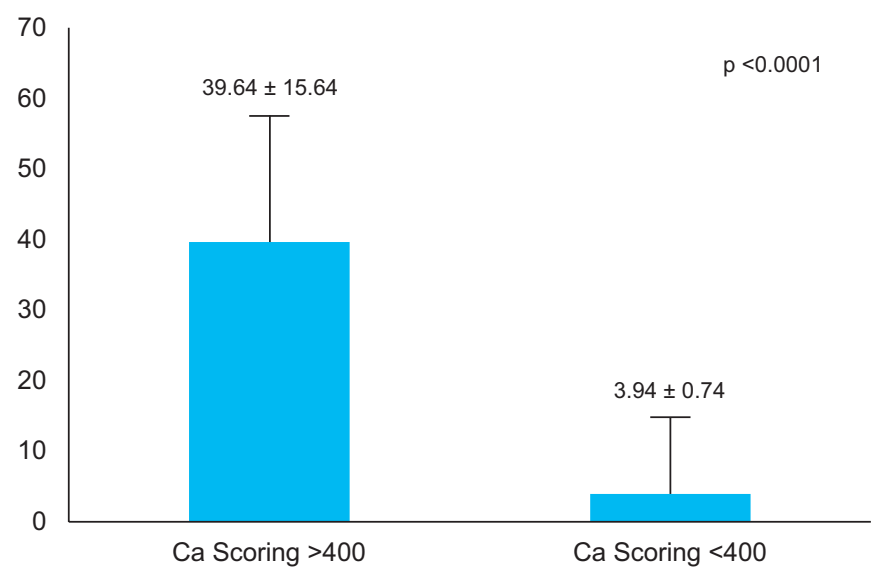

FIGURE 5. Analysis of the Syntax score. A - Patients with a high Syntax Score, $>23$; $\mathbf{B}$ - Mean values of the Syntax Score in the study groups 

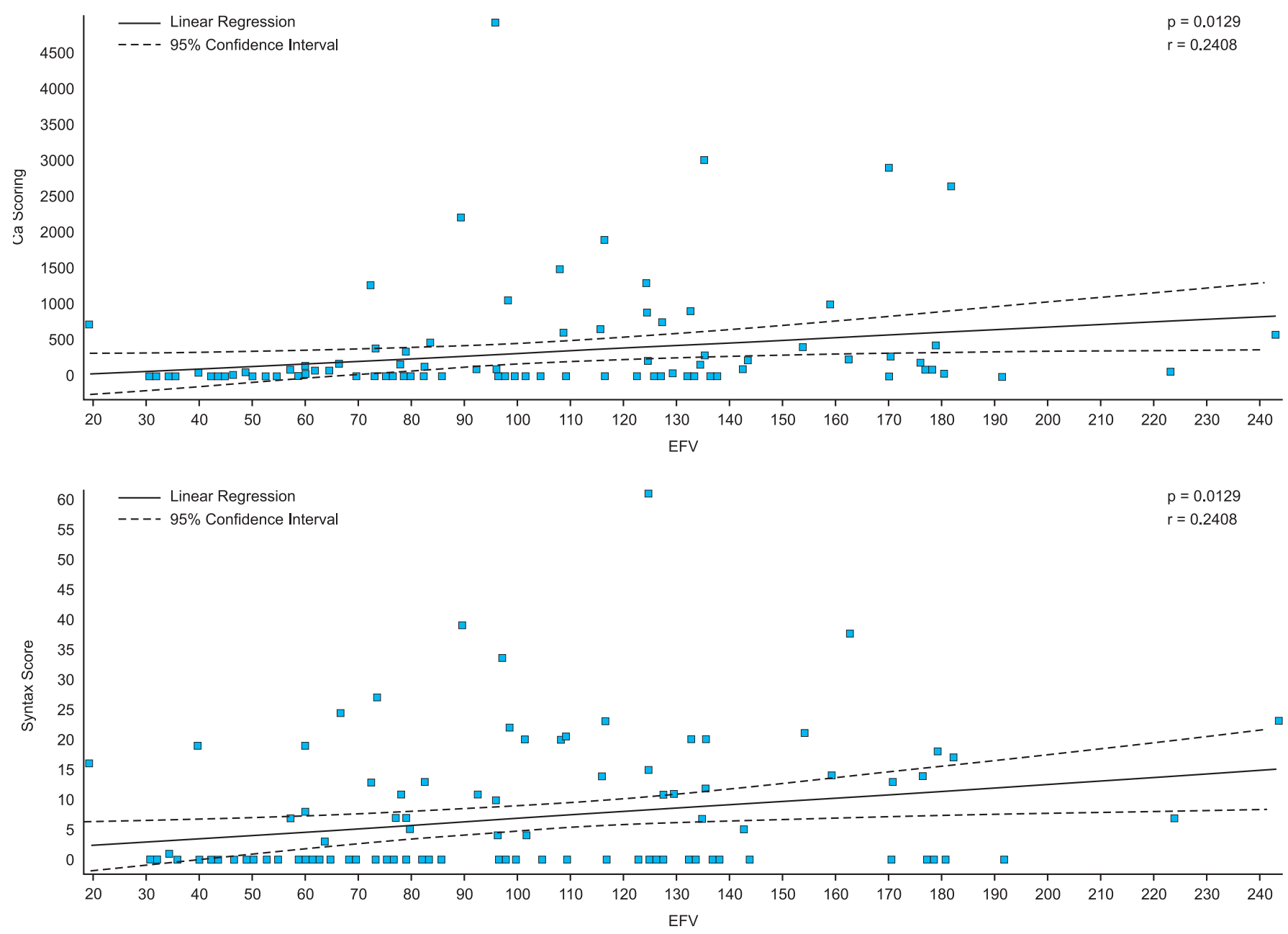

FIGURE 6. Linear regression analysis between EFV and coronary calcium score (A) and EFV and Syntax score (B)

\section{CONCLUSIONS}

In conclusion, there is a good relationship between the epicardial fat volume (measured by MSCT) and the coronary atherosclerotic burden in patients with CAD. Increased volumes of EFV were associated with other biomarkers of disease severity, such as the Coronary Calcium Score and the Syntax Score. Therefore, the epicardial fat volume could represent a new imaging-derived biomarker useful for characterization of the severity of coronary artery disease.

\section{CONFLICT OF INTEREST}

Nothing to disclose.

\section{ACKNOWLEDGEMENT}

This research was supported by the Cardio Med Medical Center, project no. CAM/2015/RD/36.

\section{REFERENCES}

1. lacobellis G, Corradi D, Sharma AM. Epicardial adipose tissue: anatomic, biomolecular and clinical relationship with the heart. Nat Clin Pract Cardiovasc Med. 2005;2(10):536-543.

2. Singh $\mathrm{N}$, Singh $\mathrm{H}$, Khanijoun $\mathrm{H}$, et al. Echocardiographic Assessment of Epicardial Adipose Tissue - A Marker of Visceral Adiposity. Mcgill J Med. 2007:10(1): 26-30.

3. lacobellis G, Bianco AC. Epicardial adipose tissue: emerging physiological pathophysiological and clinical features. Trends Endocrinol Metab. 2011;22(11):450-457

4. Zhou $Y$, Wei $Y$, Wang $X$, et al. Decreased adiponectin and increased inflammation expression in epicardial adipose tissue in coronary artery disease. Cardiovascular Diabetology. 2011;10:2

5. Fitzgibbons TP, Czech MP. Epicardial and perivascular adipose tissues and their influence on cardiovascular disease: basic mechanisms and clinical associations. J Am Heart Assoc 2014;3(2):e000582

6. Cheng KH, Chu CS, Lee KT, et al. Adipocytokines and proinflammatory mediators from abdominal and epicardial adipose tissue in patients with coronary artery disease. Int J Obes. 2008;32:268-274.

7. Talman A, Psaltis P, Cameron D, et al. Epicardial adipose tissue: far more than a fat depot. Cardiovasc Diagn Ther. 2014:4(6):416-429.

8. lacobellis G. Local and systemic effects of the multifaceted epicardial adipose tissue depot. Nat Rev Endocrinol. 2015;11(6):363-371.

9. lacobellis G, Willens HJ. Echocardiographic epicardial fat: a review of research and clinical applications. J Am Soc Echocardiogr 2009;22(12):1311-1319

10. Park EM, Choi JH, Shin IS, et al. Echocardiographic epicardial fat thikness on short term prognosis in patients with acute coronary syndrome. $J$ Cardiovasc Ultrasound. 2008;16(2):42-47. 
11. Bertaso A, Bertol D, Duncan BB, et al. Epicardial fat: definition, measurements and systematic review of main outcomes. Arq Bras Cardiol. 2013;101(1):18-28.

12. Dey D, Nakazato R, Li D, Berman DS. Epicardial and thoracic fat Noninvasive measurement and clinical implications. Cardiovasc Diagn Ther. 2012;2(2):85-93.

13. Mihl C, Loeffen D, Mathij O, et al. Automated quantification of epicardial adipose tissue (EAT) in coronary CT angiography; comparison with manual assesment and correlation with coronary artery disease. J Cardiovasc Comput Tomogr. 2014;8:215-221.

14. Coppini G, Favilla R, Marraccini P, et al. Quantification of epicardial fat by cardiac CT imaging. Open Med Inform J. 2010;4:124-135.

15. Mahabadi AA, Lehmann $\mathrm{N}$, Kalsch $\mathrm{H}$, et al. Association pf Epicardial Adipose Tissue With Progression of Coronary Artery Calcification Is More
Pronounced in the Early Phase of Atherosclerosis. JACC Cardiovasc Imaging. 2014;7(9):909-16.

16. Iwasaki K, Matsumoto $\mathrm{T}$, Aono $\mathrm{H}$, et al. Relationship between epicardial fat measured by 64-multidetector computed tomography and coronary artery disease. Clin Cardiol. 2011;34:166-71.

17. Ueno K, Anzai T, Jinzaki M, et al. Increased epicardial fat volume quantified by 64-multidetector computed tomography is associated with coronary atherosclerosis and totally occlusive lesions. Circ J. 2009;73:1927-1933.

18. Mahabadi AA, Berg MH, Lehmann N, et al. Association of epicardial fat with cardiovascular risk factors and incident myocardial infarction in the general population: the Heinz Nixdorf Recall Study. J Am Coll Cardiol. 2013;61:1388-1395. 